\title{
Permanent Draft Genome sequence for Frankia sp. strain Ccl49, a Nitrogen-Fixing Bacterium Isolated from Casuarina cunninghamiana that Infects Elaeagnaceae
}

\author{
Samira Mansour ${ }^{1 凶}$, Erik Swanson ${ }^{2}$, Zakkary McNutt², Céline Pesce ${ }^{2}$, Kelsey Harrington $^{2}$, Feseha \\ Abebe-Alele², Stephen Simpson², Krystalynne Morris², W. Kelley Thomas², and Louis S. Tisa2 ${ }^{\circledR}$ \\ 1. Suez Canal University, Ismailia, Egypt, \\ 2. University of New Hampshire, Durham, New Hampshire, USA. \\ $\triangle$ Corresponding authors: Louis S. Tisa, Mailing address: Department of Molecular, Cellular, and Biomedical Sciences, University of New Hampshire, 46 \\ College Rd., Durham, NH 03824-2617. Email: louis.tisa@unh.edu Telephone: 1-603-862-2442 Fax: 1-603-862-2621. Samira Mansour, Mailing address: Botany \\ department, Faculty of Science, Suez Canal University, Ismailia, Egypt. Email: samirarmansour@hotmail.com \\ (c) Ivyspring International Publisher. This is an open access article distributed under the terms of the Creative Commons Attribution (CC BY-NC) license \\ (https://creativecommons.org/licenses/by-nc/4.0/). See http://ivyspring.com/terms for full terms and conditions.
}

Received: 2017.07.28; Accepted: 2017.08.31; Published: 2017.09.12

\begin{abstract}
Frankia sp. strain $\mathrm{Ccl} 49$ was isolated from Casuarina cunninghamiana nodules. However the strain was unable to re-infect Casuarina, but was able to infect other actinorhizal plants including Elaeagnaceae. Here, we report the 9.8-Mbp draft genome sequence of Frankia sp. strain Ccl49 with a G+C content of $70.5 \%$ and 7,441 candidate protein-encoding genes. Analysis of the genome revealed the presence of a bph operon involved in the degradation of biphenyls and polychlorinated biphenyls.
\end{abstract}

Key words: Actinorhizal symbiosis, bioremediation, nitrogen fixation, natural products, host microbe interactions, genomes.

Soil dwelling actinobacteria of the genus Frankia form an endophytic symbiosis with actinorhizal plants, which are comprised of over 200 species from 8 angiosperm families [1, 2]. Actinorhizal plants in symbiosis with Frankia play important ecological roles as pioneer species and are used in agroforestry, land reclamation, crop protection, and soil stabilization projects [3]. Molecular phylogenetic approaches have identified four major clusters of Frankia that also follow host plant specificity groups [4-7]. Members of cluster 1 are divided into sub-cluster 1 a that are infective on Alnus and Myricaceae and sub-cluster $1 \mathrm{~b}$ strains which are infective on Allocasuarina, Casuarina and Myricacaeae. Cluster 2 represents strains infective on Coriariaceae, Datiscaceae, Dryadoideae and Ceanothus, while cluster 3 comprises strains that are infective on Colletieae, Elaeagnaceae, Gymnostoma and Myricaceae. Finally, cluster 4 groups Frankia strains isolated from actinorhizal nodules that are unable to undertake the nitrogen-fixation process (Fix) and/or re-infect their host plant causing nodulation (Nod-) and are classified as "atypical Frankia". Genomes for representatives from each cluster have been sequenced [8]. The availability of these Frankia genome databases has opened up the use of "omics" approaches. Analysis of Frankia genomes has revealed new potential in respect to metabolic diversity, natural product biosynthesis, and stress tolerance, which may aid the cosmopolitan nature of the actinorhizal symbiosis.

Several Frankia strains isolated from Casuarina nodules are unable to re-infect it, but are able to infect other actinorhizal plant genera like Elaeagnus [9, 10]. Although isolated from Casuarina nodules, these Frankia strains are classified as members of cluster 3 based on molecular phylogeny and genomes for two members of this group have been sequenced [11, 12]. Frankia sp. strain CcI49 was isolated from root nodules 
of Casuarina cunninghamiana grown on the edge of a cultivated field on side of the highway in Ismailia-Port Said, Egypt. The fresh nodules were washed, dissected into individual lobes, and surface-sterilized as described previously [13]. Each lobe was checked for sterility in sterile nutrient-rich medium. Nodules that were free from contamination were selected, dissected and homogenized, the homogenates were transferred to 100-ml screw caped bottle containing modified BAP medium for outgrowth. Hyphal outgrowth was homogenized and plated onto solid medium. After 3-4 weeks, colonies picked from the plates were homogenized and incubated in liquid medium. Surprisingly, Frankia sp. strain CcI49 produced reddish colonies, while other Frankia isolates from Casuarina do not. Frankia sp. strain CcI49 produced sporangia and spores that were smaller and narrower than normal Frankia sporangia and spores (Figure 1). Spores from Frankia sp. strain CcI49 had a high germination rate similar to Frankia strain CeI5 [14, 15]. We tested the ability of Frankia sp. strain CcI49 to re-infect actinorhizal plants. Four different actinorhizal plant species were tested to assay the plant host range and ten plants of each species were inoculated. Frankia sp. strain CcI49 was unable to infect $C$. cunninghamiana and Alnus glutinosa, but formed nodules on Elaeagnus angustifolia and Hippophäe rhamnoides. All ten of the E, angustifolia and $H$. rhamnoides plants tested formed nodules. Thus, Frankia sp. strain CcI49 had a plant-host-specificity pattern similar to Frankia sp. strains G2 and R43 $[9,10]$ from cluster 3 also isolated from Casuarina root nodules. Frankia sp. strain CcI49 genome was chosen to be sequenced for several reasons including an interesting physiology including the production of a reddish pigment, development of smaller sporangia and spores than are typical found with Frankia, and providing more information on this Frankia subcluster.

Sequencing of the draft genome of Frankia sp. strain CcI49 was performed at the Hubbard Center for Genome Studies (University of New Hampshire, Durham, NH) using Illumina technology techniques [16]. A standard Illumina shotgun library was constructed and sequenced using the Illumina HiSeq2500 platform, which generated 7,939,466 reads (260-bp insert size) totaling 1,921 MBp. The Illumina sequence data were trimmed by Trimmonatic version 0.32 [17], assembled using Spades version 3.5 [18], and ALLPaths-LG version r52488 [19]. The final draft assembly for Frankia sp. strain CcI49 consisted of 78 contigs with an $\mathrm{N}_{50}$ contig size of $282.1 \mathrm{~kb}$ and 167X coverage of the genome. The final assembled genome contained a total sequence length of $9,758,130 \mathrm{bp}$ with a $\mathrm{G}+\mathrm{C}$ content of $70.5 \%$.

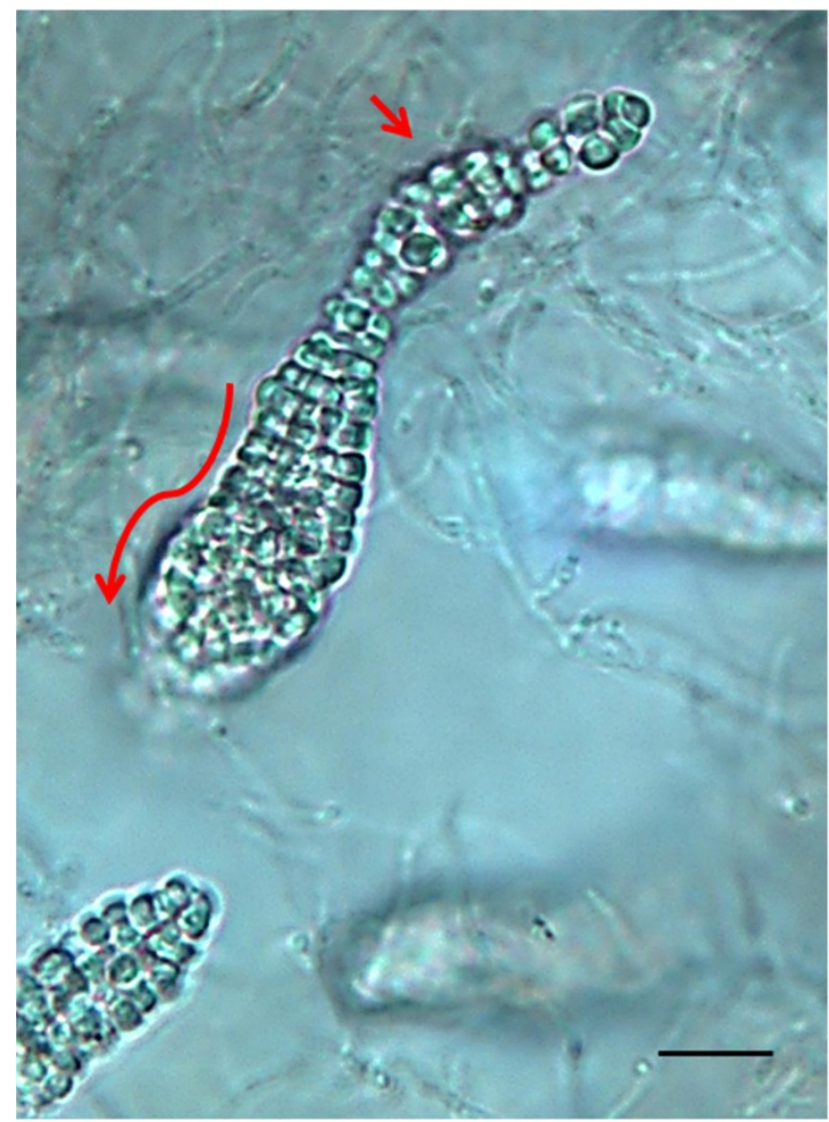

Figure 1. Photomicrograph of Franakia sp. strain Ccl49 grown in liquid culture. The elongated arrow shows the presence of a long, narrow sessile sporangium containing differentiated mature spores at distal end (short arrow). Size bar represents $32 \mu \mathrm{m}$.

The assembled Frankia sp. strain CcI49 genome was annotated via the NCBI Prokaryotic Genome Annotation Pipeline (PGAP), and resulted in 7,411 candidate protein-encoding genes, 46 tRNA and 2 rRNA regions. The genome features of Frankia sp. strain CcI49 fall outside the realm of the other cluster $1 \mathrm{~b}$ genomes, but similar to other cluster 3 isolates from Casuarina (Table 1). Phylogenetic analysis of the $23 S$ rDNA shows that Frankia sp. strain CcI49 groups with the cluster 3 strains (Figure S1). The genome size and corresponding number of CDSs were larger than the typical cluster $1 \mathrm{~b}$, but fit within those values reported for cluster 3 genomes [8]. The genome also contained a nif, 2 hup, and 1 shc operons encoding the nitrogenase and uptake hydrogenase enzymes and the hopanoid biosynthetic pathway, respectively. The operons were organized similar to those reported for Frankia cluster 3 genomes [8]. 
Table 1. Genome features of Frankia sp. strain Ccl49 and other Frankia strains isolated from Casuarina root nodules.

\begin{tabular}{|c|c|c|c|c|c|c|c|}
\hline Strain & Source & Location ${ }^{1}$ & Size $(\mathrm{Mb})$ & No. of Contigs & Frankia cluster & No. of CDS & Host Plants 2 \\
\hline CcI49 & This study & Egypt & 9.76 & 78 & 3 & 7,441 & Elaeagnaceae \\
\hline R43 & [12] & USA & 10.45 & 46 & 3 & 7,644 & Elaeagnaceae \\
\hline G2 & [11] & Guadeloupe & 9.54 & 90 & 3 & 7,790 & Elaeagnaceae \\
\hline KB5 & [23] & Australia & 5.46 & 420 & $1 b$ & 4,958 & Casuarinaceae \\
\hline $\mathrm{CcI} 3$ & [24] & USA & 5.43 & 1 & $1 b$ & 4,598 & Casuarinaceae \\
\hline $\mathrm{CeD}$ & [25] & Senegal & 5.00 & 120 & $1 b$ & 4,403 & Casuarinaceae \\
\hline Allo2 & [26] & Uruguay & 5.33 & 110 & $1 \mathrm{~b}$ & 4,838 & Casuarinaceae \\
\hline Thr & [27] & Egypt & 5.31 & 171 & $1 \mathrm{~b}$ & 4,805 & Casuarinaceae \\
\hline BMG5.23 & [28] & Tunisia & 5.27 & 167 & $1 b$ & 4,747 & Casuarinaceae \\
\hline CcI6 & [29] & Egypt & 5.39 & 138 & $1 b$ & 4,902 & Casuarinaceae \\
\hline $\mathrm{BR}$ & [30] & Brazil & 5.23 & 180 & $1 b$ & 4,777 & Casuarinaceae \\
\hline
\end{tabular}

${ }^{1}$ The source of the isolate

2 Re-infection plant host range

Analysis of the Frankia sp. strain CcI49 revealed the presence of the bph operon coding for a potential metabolic pathway involved in the degradation of biphenyl and polychlorinated biphenyls (Figure 2). The bph operon is also present in Frankia sp. strains EuI1c and EUN1f genomes [20] and was also found in the genomes of Frankia sp. strains G2 and R43 [11, 12], cluster 3 strains isolated from Casuarina root nodules. Both Frankia sp. strains CcI49 and EUN1f contained the entire bph operon, while two genes (bphA3 and $b p h H$ ) are missing in Frankia sp. EuI1c (Figure 2). The presence of the complete bph operon suggests that Frankia sp. strain CcI49 may be capable of degrading these recalcitrant xenobiotics.

Bioinformatic analysis of this genomes by the use of the AntiSMASH program [21] revealed the presence of high numbers of secondary metabolic biosynthetic gene clusters, which is consistent with previous results with other Frankia genomes including cluster $3[8,22]$. Table 2 shows a comparison of the various profiles of different Frankia strains isolated from Casuarina for these secondary metabolic biosynthetic gene clusters. The profile of Frankia sp. strain CcI49 differed from those shown by Frankia strains that are able re-infect Casuarina and was similar to the pattern exhibited by the other two cluster 3 strains (R43 and G2) isolated from Casuarina nodules. These cluster 3 genomes contained more polyketide synthase (PKS) biosynthetic clusters than the cluster $1 \mathrm{~b}$ genomes. The Frankia sp. strain CcI49 genome contained several unique clusters that had homologues in other bacteria or were completely novel.

In summary, the Frankia sp. strain CcI49 genome has revealed an interesting potential metabolic pathways and natural product profile, and serves as a representative of Frankia cluster 3. Further analysis of this genome and experimental evidence will be needed to support the predicted natural product profile and metabolic potential of Frankia sp. strain CcI49.

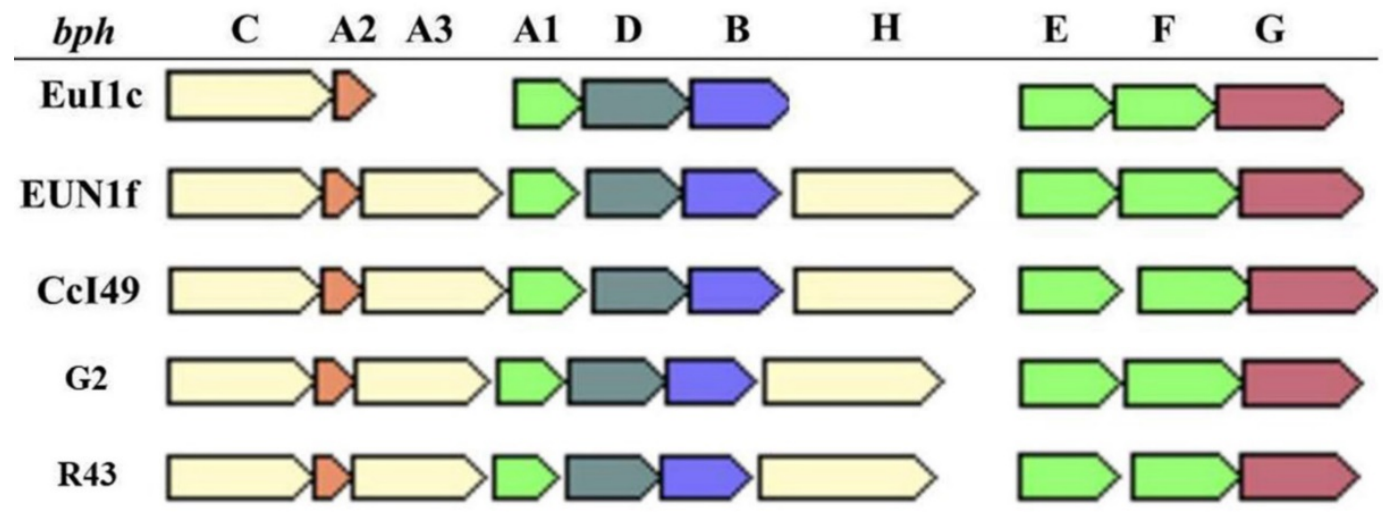

Figure 2. The bph operon is present in Frankia strains Ccl49, Eullc, G2, R43, and EUNIf. bph genes encode the following: BphA, A2 and A3, Biphenyl 2,3-dioxygenase; BphB, cis -2,3-dihydro-2,3-dihydroxybiphenyl dehydrogenase; BphC, 2,3-dihydroxybiphenyl 1,2-dioxygenase; BphD, 2-hydroxy-6-phenyl-6-oxohexa2,4-dieneoate (HOPDA) hydrolase; BphE, 2-hydroxypenta-2,4-dienoate hydratase; BphF, acylating acetaldehyde dehydrogenase; and BphG, 4-hydroxy-2-oxovalerate aldolase. 
Table 2. Biosynthetic gene clusters for natural products found in the genomes from Casuarina Frankia strains.

\begin{tabular}{|c|c|c|c|c|c|c|c|c|}
\hline Strain & Frankia Cluster & No. of Biosynthetic gene clusters ${ }^{1}$ & NRPS 2 & $\mathrm{PKS}^{3}$ & Terpene & Siderophore & Bacteriocin & Lantipeptide \\
\hline CcI49 & 3 & 42 & 6 & 17 & 3 & 1 & 2 & 5 \\
\hline R43 & 3 & 38 & 4 & 14 & 3 & 1 & 2 & 4 \\
\hline G2 & 3 & 35 & 8 & 13 & 3 & 1 & 2 & 2 \\
\hline KB5 & $1 b$ & 34 & 4 & 9 & 6 & 1 & 1 & 4 \\
\hline $\mathrm{CcI} 3$ & $1 b$ & 29 & 3 & 5 & 4 & 1 & 3 & 6 \\
\hline $\mathrm{CeD}$ & $1 b$ & 30 & 7 & 7 & 4 & 1 & 1 & 4 \\
\hline Allo2 & $1 b$ & 32 & 7 & 9 & 4 & 1 & 3 & 5 \\
\hline Thr & $1 b$ & 33 & 6 & 7 & 4 & 1 & 1 & 6 \\
\hline BMG5.23 & $1 b$ & 31 & 8 & 6 & 4 & 1 & 2 & 4 \\
\hline CcI6 & $1 b$ & 33 & 8 & 8 & 4 & 1 & 3 & 5 \\
\hline $\mathrm{BR}$ & $1 b$ & 29 & 5 & 5 & 4 & 1 & 2 & 5 \\
\hline
\end{tabular}

${ }^{1}$ Biosynthetic gene clusters were identified by the use of the AntiSMASH software [21]

2 NRPS: Nonribosomal peptide synthase

${ }^{3}$ PKS: polyketide synthase including Type I, II, III, Trans-AT, and other types

\section{Nucleotide sequence accession numbers}

This whole-genome shotgun sequence has been deposited at DDBJ/EMBL/GenBank under the accession number MOWP00000000.1. The version described in this paper is the first version, MOWP01000000.

\section{Supplementary Material}

Figure S1.

http://www.jgenomics.com/v05p0119s1.pdf

\section{Acknowledgement}

Partial funding was provided by the New Hampshire Agricultural Experiment Station. This is Scientific Contribution Number 2471. This work was supported by the USDA National Institute of Food and Agriculture Hatch 022821 (LST), Agriculture and Food Research Initiative Grant 2015-67014-22849 from the USDA National Institute of Food and Agriculture (LST), the College of Life Science and Agriculture at the University of New Hampshire-Durham and the Science and Technology Development Fund (STDF), grant No. 900, as International Cooperation Project (SM and LST). Sequencing was performed on an Illumina HiSeq2500 purchased with an NSF MRI Grant: DBI-1229361 to WK Thomas.

\section{Competing Interests}

The authors have declared that no competing interest exists.

\section{References}

1. Normand P, Benson DR, Berry AM, Tisa LS. Family Frankiaceae. In: Rosenberg E, DeLong EF, Lory S, Stackebrandt E, Thompson F, editors. The Prokaryote - Actinobacteria Berlin Heidelberg: Springer-Verlag; 2014: 339-56

2. Chaia EE, Wall LG, Huss-Danell K. Life in soil by the actinorhizal root nodule endophyte Frankia. A review. Symbiosis. 2010; 51: 201-26.

3. Benson DR, Dawson JO. Recent advances in the biogeography and genecology of symbiotic Frankia and its host plants. Physiol Plantarum. 2007; 130: 318-30.

4. Normand P, Orso S, Cournoyer B, Jeannin P, Chapelon C, Dawson J, et al. Molecular phylogeny of the genus Frankia and related genera and emendation of the family Frankiaceae. Int J Syst Bacteriol. 1996; 46: 1-9.
5. Ghodhbane-Gtari F, Nouioui I, Chair M, Boudabous A, Gtari M. 16S-23S rRNA Intergenic Spacer Region Variability in the Genus Frankia. Microb Ecol. 2010; 60: 487-95

6. Nouioui I, Ghodhbane-Gtari F, Beauchemin NJ, Tisa LS, Gtari M. Phylogeny of members of the Frankia genus based on gyrB, nifH and glnII sequences. Anton Leeuw Int J G. 2011; 100: 579-87.

7. Gtari M, Tisa LS, Normand P. Diversity of Frankia Strains, Actinobacterial Symbionts of Actinorhizal Plants. In: Aroca R, editor. Symbiotic Endophytes. 2013: 123-48.

8. Tisa LS, Oshone R, Sarkar I, Ktari A, Sen A, Gtari M. Genomic approaches toward understanding the actinorhizal symbiosis: an update on the status of the Frankia genomes. Symbiosis. 2016; 70: 5-16.

9. Zhang Z, Lopez MF, Torrey JG. A Comparison of Cultural-Characteristics and Infectivity of Frankia Isolates from Root-Nodules of Casuarina Species. Plant Soil. 1984; 78: 79-90.

10. Diem HG, Gauthier D, Dommergues YR. Isolation of Frankia from Nodules of Casuarina equisetifolia. Can J Microbiol. 1982; 28: 526-30.

11. Nouioui I, Gtari M, Goker M, Ghodhbane-Gtari F, Tisa LS, Fernandez MP, et al. Draft Genome Sequence of Frankia Strain G2, a Nitrogen-Fixing Actinobacterium Isolated from Casuarina equisetifolia and Able To Nodulate Actinorhizal Plants of the Order Rhamnales. Genome Announc. 2016; 4: e00437-16.

12. Pujic P, Bolotin A, Fournier P, Sorokin A, Lapidus A, Richau KH, et al. Genome Sequence of the Atypical Symbiotic Frankia R43 Strain, a Nitrogen-Fixing and Hydrogen-Producing Actinobacterium. Genome Announc. 2015; 3: e01387-15.

13. Mansour SR, Dewedar A, Torrey JG. Isolation, Culture, and Behavior of Frankia Strain HFPCgI4 from Root Nodules of Casuarina glauca. Bot Gaz. 1990; 151: 490-6.

14. Tzean SS, Torrey JG. Spore Germination and the Life Cycle of Frankia Invitro. Can J Microbiol. 1989; 35: 801-6.

15. Mansour SR, Torrey JG. Frankia Spores of Strain Hfpcgi4 as Inoculum for Seedlings of Casuarina glauca. Can J Bot. 1991; 69: 1251-6.

16. Bennett S. Solexa Ltd. Pharmacogenomics. 2004; 5: 433-8.

17. Bolger AM, Lohse M, Usadel B. Trimmomatic: a flexible trimmer for Illumina sequence data. Bioinformatics. 2014; 30: 2114-20.

18. Nurk S, Bankevich A, Antipov D, Gurevich AA, Korobeynikov A, Lapidus A, et al. Assembling single-cell genomes and mini-metagenomes from chimeric MDA products. Journal of computational biology : a journal of computational molecular cell biology. 2013; 20: 714-37.

19. Gnerre S, MacCallum I, Przybylski D, Ribeiro FJ, Burton JN, Walker BJ, et al. High-quality draft assemblies of mammalian genomes from massively parallel sequence data. P Natl Acad Sci USA. 2011; 108: 1513-8.

20. Rehan M, Swanson E, Tisa LS. Frankia as a Biodegrading Agent. In: Dhanasekaran D, Jiang Y, editors. Actinobacteria - Basics and Biotechnological Applications. Croatia: In Tech Open Access 2016: 271-90.

21. Blin K, Wolf T, Chevrette MG, Lu XW, Schwalen CJ, Kautsar SA, et al. antiSMASH 4.0-improvements in chemistry prediction and gene cluster boundary identification. Nucleic Acids Res. 2017; 45: W36-W41.

22. Udwary DW, Gontang EA, Jones AC, Jones CS, Schultz AW, Winter JM, et al. Significant Natural Product Biosynthetic Potential of Actinorhizal Symbionts of the Genus Frankia, as Revealed by Comparative Genomic and Proteomic Analyses. Appl Environ Microb. 2011; 77: 3617-25.

23. Pesce C, Swanson E, Simpson S, Morris K, Thomas WK, Tisa LS, et al. Draft Genome Sequence for Frankia sp. Strain KB5 Isolated from the Root Nodules of Casuarina equisetifolia. . J Genomics. 2017; 5: 64-7

24. Normand P, Lapierre P, Tisa LS, Gogarten JP, Alloisio N, Bagnarol E, et al. Genome characteristics of facultatively symbiotic Frankia sp strains reflect host range and host plant biogeography. Genome research. 2007; 17: 7-15.

25. Ngom M, Oshone R, Hurst SGt, Abebe-Akele F, Simpson S, Morris K, et al. Permanent Draft Genome Sequence for Frankia sp. Strain CeD, a 
Nitrogen-Fixing Actinobacterium Isolated from the Root Nodules of Casuarina equistifolia Grown in Senegal. Genome announcements. 2016; 4: e00265-16.

26. Oshone R, Ngom M, Abebe-Akele F, Simpson S, Morris K, Sy MO, et al Permanent Draft Genome Sequence of Frankia sp. Strain Allo2, a Salt-Tolerant Nitrogen-Fixing Actinobacterium Isolated from the Root Nodules of Allocasuarina. Genome announcements. 2016; 4: e00388-16.

27. Hurst SGt, Oshone R, Ghodhbane-Gtari F, Morris K, Abebe-Akele F, Thomas WK, et al. Draft Genome Sequence of Frankia sp. Strain Thr, a Nitrogen-Fixing Actinobacterium Isolated from the Root Nodules of Casuarina cunninghamiana Grown in Egypt. Genome announcements. 2014; 2: e00493-14.

28. Ghodhbane-Gtari F, Hurst SGt, Oshone R, Morris K, Abebe-Akele F, Thomas WK, et al. Draft Genome Sequence of Frankia sp. Strain BMG5.23, a
Salt-Tolerant Nitrogen-Fixing Actinobacterium Isolated from the Root Nodules of Casuarina glauca Grown in Tunisia. Genome announcements. 2014; 2: $\mathrm{e} 00520-14$

29. Mansour SR, Oshone R, Hurst SGt, Morris K, Thomas WK, Tisa LS. Draft Genome Sequence of Frankia sp. Strain CcI6, a Salt-Tolerant Nitrogen-Fixing Actinobacterium Isolated from the Root Nodule of Casuarina cunninghamiana. Genome announcements. 2014; 2: e1205-13.

30. D'Angelo T, Oshone R, Abebe-Akele F, Simpson S, Morris K, Thomas WK, et al. Permanent Draft Genome Sequence of Frankia sp. Strain BR, a Nitrogen-Fixing Actinobacterium Isolated from the Root Nodules of Casuarina equisetifolia. Genome announcements. 2016; 4: e01000-16. 